\title{
Nanocarriers of Antisense Oligonucleotides in Diabetes
}

Novella Rapini, ${ }^{1, a}$ Nunzio Bottini ${ }^{1, b}$ and Massimo Bottini ${ }^{2, *}$

\begin{abstract}
Antisense oligonucleotides are short strands of deoxyribonucleotides that are complementary to specific encoding mRNA sequences and can block gene expression. Their potential use as therapeutic and gene validation tools has elicited great interest. However, poor intracellular delivery into several tissues in living animals currently limits the range of in vivo applications of antisense oligonucleotides. Innovative solutions to this problem are coming from nanomedicine, a discipline recently born from the marriage of nanotechnology and medicine. Through the use of nanotechnology-derived composites(nanocarriers), nanomedicine helps traditional drugs to avoid the body's defenses that the drug encounters following its systemic administration. In this chapter we will describe diabetes-relevant applications of nanocarriers as
\end{abstract}

\footnotetext{
${ }^{1}$ La Jolla Institute for Allergy \& Immunology, 9420 Athena Circle, La Jolla, CA, 92037, USA.

${ }^{a}$ E-mail: nrapini@liai.org

bE-mail: nunzio@liai.org

${ }^{2}$ Sanford-Burnham Medical Research Institute, 10901 North Torrey Pines Road, La Jolla, CA, 92037, USA; E-mail: mbottini@sanfordburnham.org

*Corresponding author

List of abbreviations after the text.
} 
delivery systems for antisense oligonucleotides. We divided the chapter in two sections. In the first section we will 1) describe the modes of action of antisense oligonucleotides and the chemical modifications that have been developed to ameliorate resistance to nucleases, enhance affinity and potency, and reduce toxicity; 2) the biological barriers encountered by the antisense oligonucleotides following their systemic administration; 3 ) the arsenal of solutions that nanomedicine can offer to enable the safe delivery of antisense oligonucleotides to the target site. In the second section, we will review the antisense oligonucleotides and delivery systems that have been developed as novel therapeutic weapons against diabetes. This includes research from our laboratory focusing on achieving targeted delivery of antisense oligonucleotides into $\mathrm{T}$ cells for therapy of Type 1 Diabetes.

\section{INTRODUCTION}

Antisense oligomers (ASOs) are short strands (typically 20bp in length) of deoxyribonucleotides that, upon intracellular delivery, hybridize to specific mRNAs and cause reduced expression of the encoded proteins (Chan et al. 2006). ASOs represent a powerful strategy for gene validation and for the cure of diseases associated to dysregulated protein expression, such as autoimmune diseases and cancer. Several chemical modifications of ASOs have been developed which dramatically improved their resistance to nucleases, circulation half-life, affinity for mRNA and silencing potency compared to first-generation oligos. However, a persisting limitation to the use of ASOs in vivo is their low spontaneous cell-permeability, especially in certain cell types. Several approaches based on physical methods (e.g., electroporation) or the use of delivery agents (e.g., viral vectors and cationic liposomes) are available to ensure efficient introduction of ASOs into cells in culture. However, comparable systems for in vivo application are still in the early stages of development, and the vast majority of ASOs undergoing clinical trials for FDA-approval are topically or systemically administered in "free" form. There is a critical need for novel delivery systems to enable the safe delivery of ASOs to targeted cells and tissues after systemic administration. Innovative solutions in this field are coming from nanomedicine, a discipline recently born from the marriage of nanotechnology and medicine. Through the use of nanotechnology-derived composites (nanocarriers), nanomedicine helps traditional drugs avoid the biological and biophysical barriers the drug encounters following its systemic administration. In this chapter we will describe diabetes-relevant applications of nanocarriers as delivery systems for ASOs. We divided the 
chapter in two sections. In the first section we will summarize the modes of action of ASOs and the chemical modifications that have been developed to ameliorate the resistance to nucleases, enhance affinity and potency, and reduce toxicity ( $\S 2.1$.), the biological barriers encountered by ASOs following their systemic administration (§ 2.2. and § 2.3.) and the range of solutions that nanomedicine can offer to enable the safe delivery of ASOs to the target site $(\S 2.4$. and $\S 2.5)$. In the second section, we will review ASOs and the delivery systems that have been developed for applications in the diabetes field. This includes research from our laboratory focused on achieving targeted delivery of ASOs into T cells for therapy of Type 1 Diabetes.

\section{ASOs AND NANOMEDICINE}

\subsection{Nanodrugs for ASO Delivery \& Targeting}

Oligonucleotide-based therapy can be based on the use of ASOs, small interference RNA (siRNA) or aptamers. Each type of drug blocks the expression of proteins by means of different intracellular mechanisms. In this chapter we will focus our attention on ASOs as a weapon against diabetes and on nanotechnology-based approaches that have been developed or are under development to improve their potency and delivery.

ASOs are short (typically 20bp in length) single-stranded deoxyribonucleotide analogues that hybridizes with the complementary mRNA via Watson-Crick base pairing. ASOs are excellent tools for target validation and gene function studies, but also highly appealing as therapeutic strategy to selectively suppress the expression of diseaserelated proteins. It is worthy of notice that the single strand structure of ASOs makes them particularly suited for in vivo immunological applications since at least some types of ASOs are believed to be less prone to elicit "stress" immune responses through receptors on innate immune cells (such as toll-like receptor 3, TLR-3, which binds double helical nucleic acids). Formation of the ASO-mRNA heteroduplex can result in inhibition of the target gene expression by various mechanisms, depending on the chemical make-up (generation) of the ASO (Fig. 1). Despite usually showing much lower potency than siRNAs, unmodified DNA ASOs can operate in a way similar to siRNA by activating endogenous ribonuclease $\mathrm{H}$ (RNase $\mathrm{H}$ ) which recognizes the DNA-RNA heteroduplex and cleaves the RNA strand leaving intact the ASO. However, the use of unmodified ASOs has been largely unsuccessful because of their susceptibility to degradation by nucleases and their inability to penetrate mammalian 


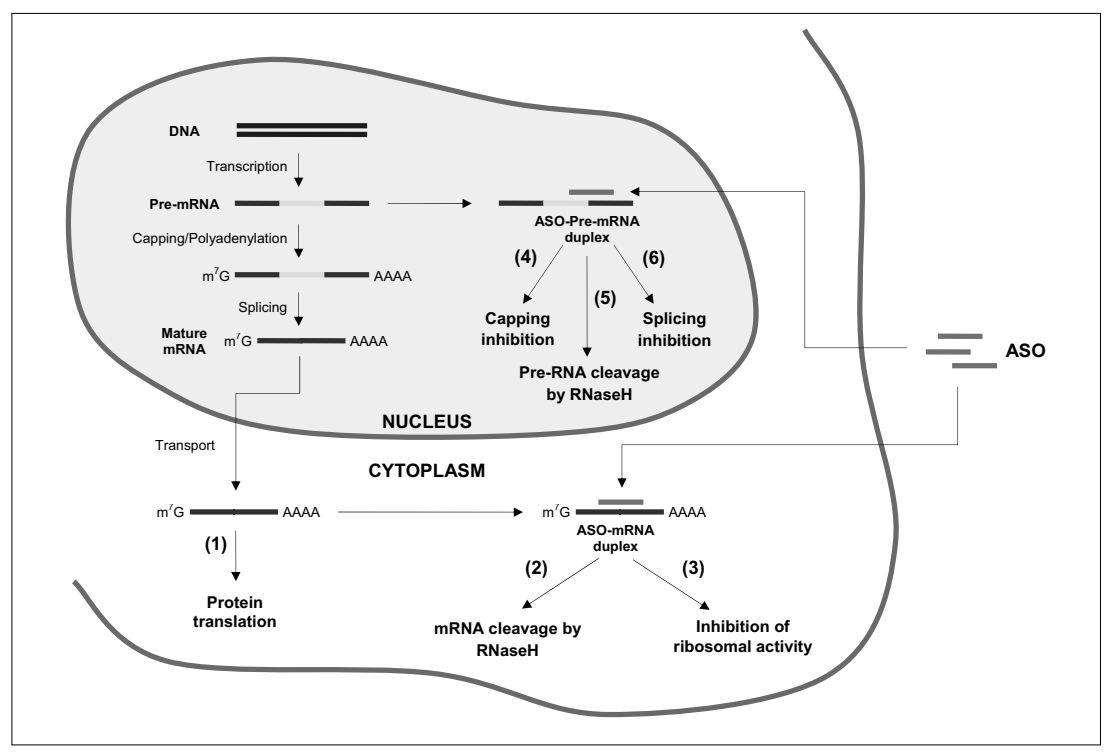

Fig. 1. Mechanism and location of action of ASOs. In absence of ASOs, mRNAs are normally translated into functional proteins (1). ASOs that are delivered into cells can hybridize with either the mRNA (in the cytoplasm) or the Pre-mRNA (in the nucleus). In the cytoplasm, the formation of the ASO-mRNA hetero-duplex can lead either to activation of RNaseH (2) or to inhibition of ribosomal activity (3). Alternatively, the ASO can migrate into the nucleus, and block the maturation of mRNA at different level of the process (4-6). The mechanism and location of action of an ASO depend on its chemical make-up and on the technique used to deliver it into the cells. Independently of mechanism and location, the action of an ASO will finally result in protein expression knock-down.

cells in culture. Various chemical modifications of the phosphodiester bond and/or the ribose sugar have been developed to decrease nuclease cleavage and increase the biostability and potency of the ASOs (Fig. 2). For instance, phosphorothioate-modified (first generation) ASOs have one of the non-bridging oxygen atoms in the phosphodiester bond replaced by a sulphur atom, whereas phosphorodiamidate morpholino-modified (third generation) ASOs are non-charged oligomers in which the phosphodiester bond and the ribose sugar are replaced by a phosphorodiamidate linkage and a six-membered morpholino ring, respectively. Morpholino ASOs are extremely suitable for in vivo applications because they are highly resistant to nucleases and proteases in biological fluids.

Despite these improvements in chemical make-up, the use of ASOs for in vivo knock-down of gene expression is still significantly limited by their poor intracellular delivery. Novel solutions to enhance ASO delivery are needed in order to fully exploit the potential of ASOs for diagnosis and therapy of human diseases. Some of these solutions are emerging from the 
Zero generation

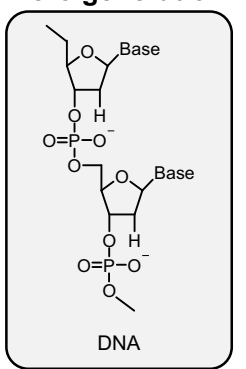

First generation

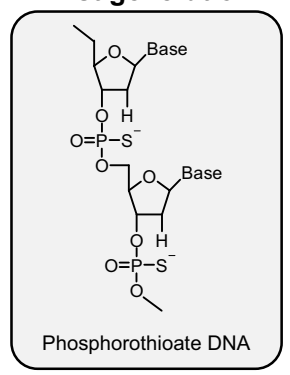

Second generation

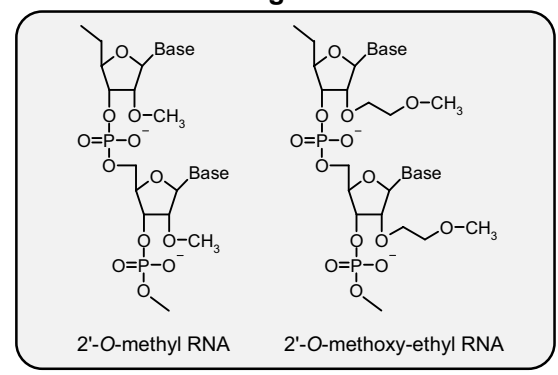

Third generation

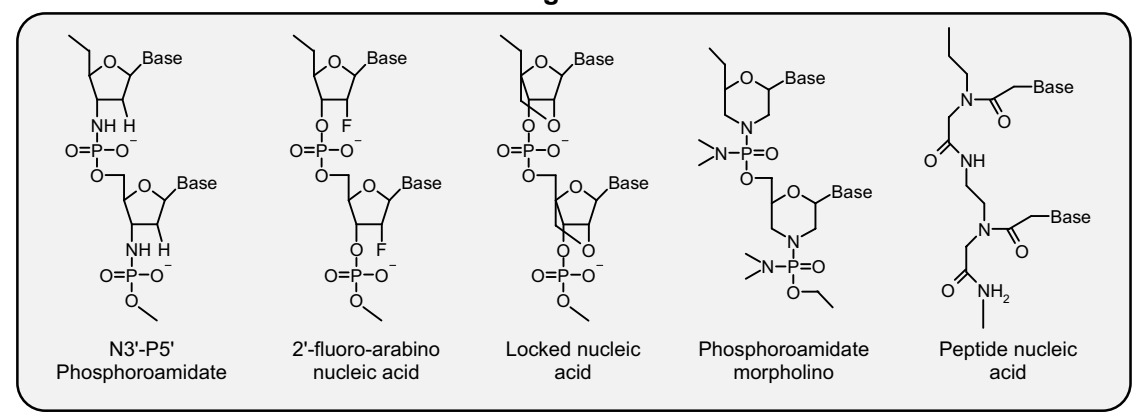

Fig. 2. Chemical modifications of antisense oligonucletides. Chemical modifications (generations) developed to decrease nuclease cleavage and increase the biostability and potency of the ASOs.

field of nanotechnology. Nanotechnology is the science of manipulating the matter at the atomic and molecular level to obtain materials with specifically enhanced chemical and physical properties. The application of nanotechnology in medicine (nanomedicine) aims at developing multifunctional nanoparticles (nanodrugs) for delivering pharmaceutical, therapeutic, and diagnostic agents to the target sites with high efficacy and specificity. Compared to traditional approaches, the ultimate promise of nanodrugs is to achieve therapeutic and diagnostic goal, using less amounts of drugs and with less or no side effects. A nanodrug can be thought as a tripartite structure composed by a nanotechnology-derived nanoparticle (nanocarrier), loaded with therapeutic and diagnostic payloads (cargos, for instance the ASOs) and with "helper" agents (enhancers) which enable the nanodrug to resist and/or penetrate the biological barriers encountered following in vivo administration (Fig. 3). 


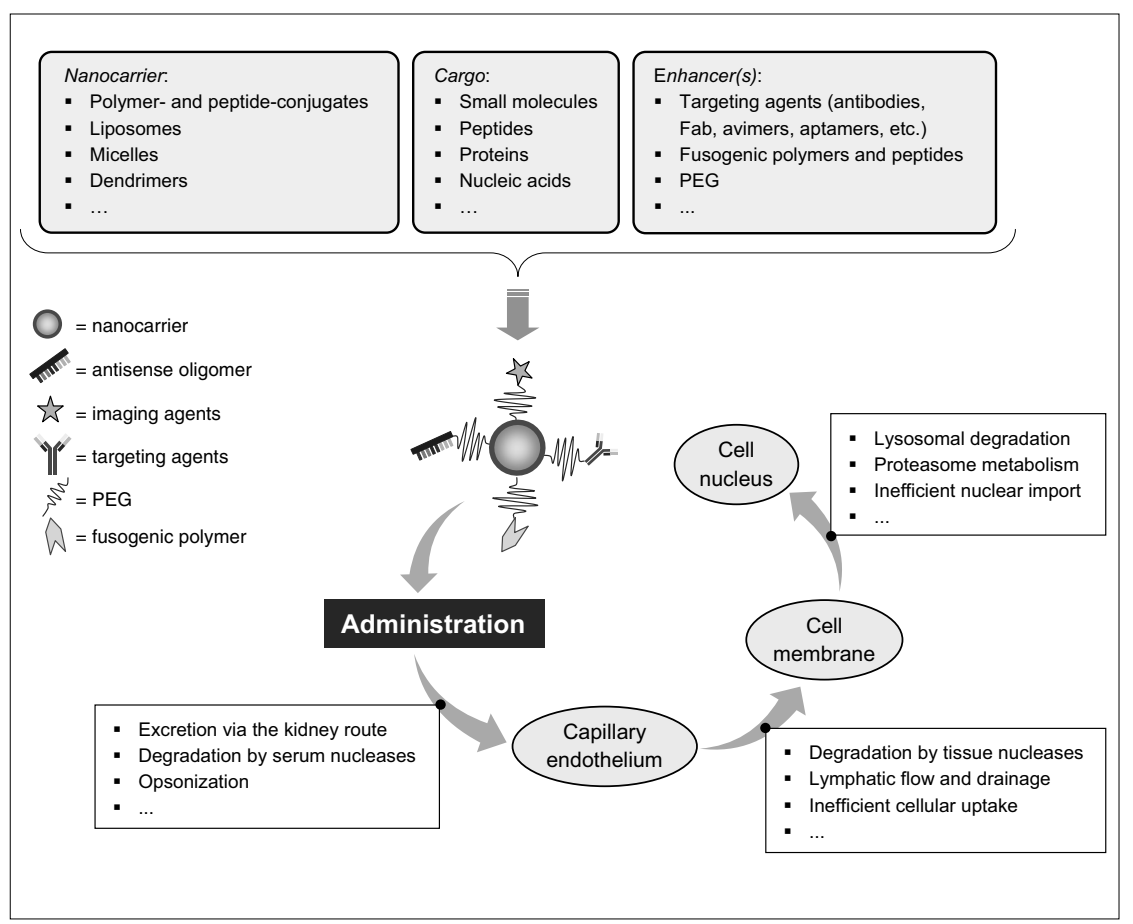

Fig. 3. The nanotechnology approach. Multifunctional nanoparticles (nanodrugs) are tripartite structures composed by a nanocarrier loaded with cargos (diagnostic and therapeutic payloads) and enhancers (targeting, permeation and imaging agents). Enhancers allow the nanoparticle to reach the site of interest avoiding the biological and biophysical barriers encountered following the systemic administration.

\subsection{The Biological Barriers: RES, Extravasation \& Diffusion}

To address the challenge of delivery ASOs to the site of action (for instance the nucleus of a specific cell subpopulation) in vivo with nanotechnology, it is necessary to combine the rational design of a nanodrug with the fundamental understanding of the several biological and physical barriers, which the nanodrug will encounter after injection in a living organism (Fig. 3).

Following their administration, nanodrugs with molecular weights smaller than $5 \mathrm{kDa}$ (such as "free" ASOs, and polymer- and peptideconjugates) may be rapidly excreted via the kidney route (Brenner et al. 1976). Larger nanodrugs are not filtered as efficiently through the kidney 
glomerular system and they are often significantly bound by opsonins in the bloodstream such as immunoglobulins, complement components and other serum proteins. This can be followed by internalization by professional phagocytic cells of the reticulo endothelial system (RES), and delivery into lysosomal compartments where they are subjected to degradation (Mosser and Edwards 2008). Cells of RES include circulating monocytes and tissue macrophages, including specialized ones such as the Kupffer cells in the liver. Liver and spleen are enriched with RES cells and characterized by a highly fenestrated vascular endothelium with gaps that allow the transit of particles up to $200 \mathrm{~nm}$ in diameter (Scherphof 1991). Thus it is not surprising that those organs are the major accumulation sites of nanodrugs. Strategies to avoid uptake by the RES are mainly based on passivation of the nanodrug surface with polyethylene glycol (PEG) chains (Juliano et al. 2008).

While circulating in the blood stream, the nanodrugs must pass the endothelial cells lining the vascular lumen in order to target specific tissue parenchymal cells. In general, nanodrugs with lateral dimension smaller than approximately $5 \mathrm{~nm}$ readily egress the capillary endothelium via paracellular routes involving imperfections in the cell junctions, whereas larger nanodrugs are able to pass across the capillary vasculature only in certain specialized tissues (for instance spleen and liver) and in defective tissues (such as tumor vessels and pancreatic islet blood vessels in diabetic patients). The tumor microenvironment is also characterized by a scarce lymphatic drainage, which, along with the leakiness of the vascular system, results in the so-called enhanced permeability and retention (EPR) effect. Several studies have been shown that larger nanodrugs (nanoparticles) accumulate in tumor tissues due to the EPR effect in higher amount than smaller nanodrugs ("free" ASOs and conjugates). However, the latter may be a better therapeutic choice if used in combination with anti-angiogenic agents, which are intended to "normalize" the tumor tissue vasculature.

Once extravasated from the blood vessels, the nanodrugs must diffuse into the extracellular matrix which is composed by a dense meshwork of proteins that might bind the nanodrugs and tends to block their diffusion (Juliano 2007). Once in the extracellular matrix, the nanoparticle must target some or all tissue cells, be internalized, and either diffuse in the cytoplasm or reach the nucleus. The process of cellular internalization of a nanoparticle is usually energy-dependent and follows one of the following pathways: 1) clathrin-dependent endocytosis, 2) the lipid raft pathway, 3) the caveolar pathway, 4) phagocytosis, and 5) pinocytosis (Fig. 4). Even if more rarely, nanoparticles may also diffuse through the plasma membrane and be internalized by an energy-independent process; an example of this is given by certain kind of functionalized carbon nanotubes that behave as nano-needles and migrate into the cytoplasm by puncturing the plasma 


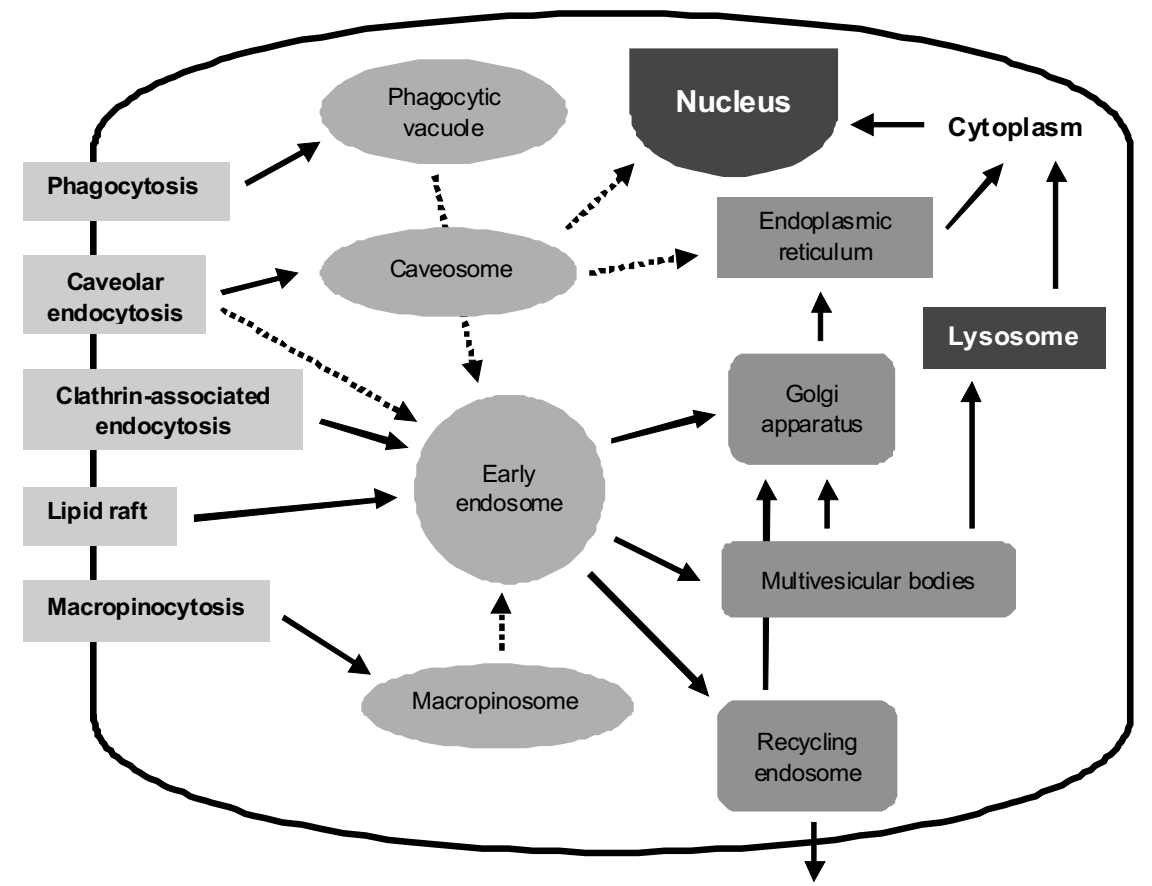

Fig. 4. Endocytic pathways and trafficking routes. Cells uptake macromolecules and particles by a process called endocytosis. Phagocytosis involves the ingestion of large substances by "professional" cells (phagocytes). The substances are ingested in the phagocytic vacuoles and transported in the lysosomes. Macropinocytosis is a clathrin-independent mechanism through membrane ruffling and responsible for the intracellular delivery of large amount of solutes and fluids through large vesicles of heterogeneous dimensions (micropinosomes). Clathrin-mediated endocytosis is generated by the invagination and pinching off of regions of the plasma membranes (coated pits) rich of a fibrous protein (clathrin) which together with a smaller polypeptide form a basketlike network of hexagon and pentagons on the surface of pits and intracellular vesicles. The endocytic vesicles subsequently lose their coats and deliver their content to the sorting endosomes. Three distinct pathways have been identified that sort the endosomes and bring their content to either the late endosomes/lysosomes for degradation, or the plasma membrane through recycling endosomes, or the endoplasmic reticulum through the trans-Golgi network and the Golgi apparatus (retrograde transport). Advances in the design of specific molecular inhibitors enabled to elucidate clathrinindependent pathways stimulated via certain microdomains of the plasma membrane, caveolae and lipid rafts, enriched in cholesterol and glycosphingolipids. Dark arrows represent established intracellular routes, whereas those represented by dotted arrows are supported by more preliminary evidence.

membrane without triggering any of the energy-dependent pathways described in Fig. 4 (Kostarelos et al. 2007). Following cellular uptake, the nanodrug is trafficked into a variety of intracellular compartments and, finally, either degraded in the lysosomes, or transported to a specific site. 


\subsection{Cutting Edge Area of Research: Active Targeting}

Traditional drugs passively accumulate in tissues by mechanisms that exploit physical and/or chemical gradients or fenestrations in blood vessels. Even if those mechanisms could target particular tissues with high efficiency, they cannot be used to carry traditional drugs (or nanodrugs) to specific sites (active targeting). Cell-specific targeting is a form of active targeting where particles are transported into a specific cell subpopulation through receptor-mediated endocytosis (RME). Here, the drug is functionalized with agents (ligands) able to recognize specific targets on the surface of cells (antigens and receptors). Efficient cell-specific targeting is based on high target specificity; the antigen/receptor must be expressed only on the cell population of interest, and on the selectivity and efficacy of the targeting agent. The ligand could bind the target with high affinity and/or avidity - a way to enhance targeting efficacy through simultaneous interactions with low affinity. Traditional drugs are usually conjugated to a single ligand, monovalent structure, whereas nanodrugs are able to carry multiple ligands thereby enhancing targeting avidity.

A recent report showed that gold and silver spherical nanoparticles functionalized with Herceptin and having a diameter falling in a $25 \mathrm{~nm}-$ centered narrow range of values can be internalized by breast cancer cells through HerB2-mediated endocytosis. Nanoparticles with diameters not in that range of values were not internalized (Jiang et al. 2008). Since HerB2 is a normally internalization-impaired receptor, these results suggest that modulation of the physico-chemical properties of the nanocarrier can induce RME after engagement of surface receptors even the ones that are naturally less prone to post-engagement internalization.

\subsection{The Arsenal of Nanocarriers}

An extremely wide range of nanocarriers have been used as backbone for the fabrication of nanodrugs. Nanocarriers can be classified by their dimension (for instance into conjugates and nanoparticles), their chemical composition (polymeric, amphiphile-based, organic, inorganic), their shape and geometry (spherical, cylindrical, toroidal, shell, tree-like, wormlike), or their physical properties (magnetic, luminescent, flexible, soft, degradable). Here we will give a quick overview of the most commonly used nanocarriers, without following a specific classification.

Polymers are among the most widely investigated materials for the fabrication of novel and high-performance nanocarriers. In the simplest application, the nanocarrier is a polymeric chain directly conjugated to 
the ASO to ameliorate its solubility and/or help its release from the endolysosomal compartments. Otherwise, biodegradable polymers and block co-polymers have been used to fabricate nanoparticles encapsulating (by electrostatic interaction) the ASOs. The oligo is then released by diffusion or erosion of the polymeric lattice in a predictable manner (Khan et al. 2004).

Amphiphile-based nanoparticles are the most widely used nanocarriers and, in function of their architecture, they are classified into liposomes and micelles. Liposomes are FDA-approved spherical structures composed by several concentric lipid bilayers with a water core (water-in-water), and are used to deliver a wide range of drugs. Cationic liposomes are the most commonly used delivery systems for first and second generation ASOs. Micelles are self-assembled spherical lipid monolayers with a hydrophobic core and a hydrophilic outer shell (oil-in-water) and are under clinical evaluation as carriers for several water insoluble drugs (including phosphorodiamidate morpholino-modified ASOs) (DeLong et al. 1999).

Organic nanoparticles such as dendrimers, peptide- and/or proteinconjugates, and carbon nanotubes have been also used to deliver ASOs. Dendrimers are tree-like structures whose synthesis is based on the growth of branched synthetic polymers (Yoo and Juliano 2000), whereas peptide- and protein-nanoparticles are fabricated by conjugating the ASO directly to peptides or proteins in order to address solubility problems and/or facilitate their intracellular internalization and trafficking (Kang et al. 2008). Carbon nanotubes (NTs) are hollow graphitic tubes, about 1 $\mathrm{nm}$ in diameters and hundreds of nanometers long, which are able to emit light in the near infrared region and are characterized by a high surface-tovolume ratio. Our research group has recently used NTs to deliver ASOs into T cells in vitro (Delogu et al. 2009).

Inorganic nanoparticles have been extensively studied as nanocarriers because of their peculiar physico-chemical properties which can be exploited to fabricate multi-functional nanoparticles. Among the inorganic nanoparticles reported as nanocarriers for ASOs, gold nanoparticles are often preferred because of their easy synthesis and uniform size (Rosi et al. 2006; Rink et al. 2010). Quantum dots are inorganic semiconducting nanocrystals that are able to emit in a narrow range of wavelengths which can be controlled since it depends on the composition and dimension of the particle (Jia et al. 2007). 


\section{ANTISENSE OLIGONUCLEOTIDE THERAPY IN DIABETES}

ASOs are under development for therapeutic applications in the type 1 and type 2 diabetes (T1D and T2D). However use of nanocarriers for the delivery of those ASOs is at its infancy and few studies has been reported (Table 1).

Table 1. ASOs for therapeutic applications in diabetes. Published studies about the use of ASOs for therapeutic applications applications in the type 1 and type 2 diabetes.

\begin{tabular}{|c|c|c|c|c|}
\hline \begin{tabular}{|c|} 
ASO \& \\
Generation
\end{tabular} & Target & Route & Nanocarrier & Reference \\
\hline $\begin{array}{l}\text { iCo-007 } \\
\text { second }\end{array}$ & c-Raf kinase & $\begin{array}{c}\text { intravitreous } \\
\text { injection in mice }\end{array}$ & none & Hnik et al. 2009 \\
\hline \begin{tabular}{|c|}
$\begin{array}{c}\text { ISIS113715 } \\
\text { second }\end{array}$ \\
\end{tabular} & PTP-1B & $\begin{array}{c}\text { intraperitoneal } \\
\text { injection in mice }\end{array}$ & none & Waring et al. 2003 \\
\hline \begin{tabular}{|c|} 
ISIS113715 \\
third (LNA)
\end{tabular} & PTP-1B & $\begin{array}{l}\text { intraperitoneal } \\
\text { injection in mice }\end{array}$ & none & Koizumi et al. 2007 \\
\hline $\begin{array}{c}\text { ISIS113715 } \\
\text { second }\end{array}$ & PTP-1B & $\begin{array}{c}\text { interscapular } \\
\text { injection in } \\
\text { monkeys }\end{array}$ & none & Swarbrick et al. 2009 \\
\hline - & $\begin{array}{c}\text { 11ßhydroxysteroid } \\
\text { dehydrogenase }\end{array}$ & $\begin{array}{l}\text { intraperitoneal } \\
\text { injection in mice }\end{array}$ & none & Bhat et al. 2008 \\
\hline second & $\begin{array}{l}\text { glucocorticoid } \\
\text { receptor }\end{array}$ & $\begin{array}{l}\text { intraperitoneal } \\
\text { injection in mice }\end{array}$ & none & Liang et al. 2005 \\
\hline third (LNA) & PPAR $\gamma$ & in vitro & $\begin{array}{c}\text { Lipofectamine } \\
2000\end{array}$ & Tachibana et al. 2007 \\
\hline second & $\begin{array}{c}\text { CD80, CD86, } \\
\text { CD40 }\end{array}$ & $\begin{array}{c}\text { subcutaneous } \\
\text { injection in mice }\end{array}$ & $\begin{array}{c}\text { PROMAXX } \\
\text { microspheres }\end{array}$ & Phillips et al. 2008 \\
\hline- & eGFP & in vitro & $\begin{array}{c}\text { gold } \\
\text { nanoparticles }\end{array}$ & Rink et al. 2010 \\
\hline second & PTPN22 & in vitro & $\begin{array}{c}\text { carbon } \\
\text { nanotubes }\end{array}$ & Delogu et al. 2009 \\
\hline
\end{tabular}

"ASO's generation was not specified in the Materials and Methods section.

\section{1 iCo-007 for Diabetic Retinopathy and Diabetic Macular Edema}

Hyperglycemia in diabetic patients alters hemodynamics of the retinal vasculature and causes chronic hypoxia, which in turns results in the upregulation of growth factors and in pathology such as neovascularization, vascular permeability, macular edema, etc. The inhibition of the kinase c-Raf is envisaged as an effective therapeutic strategy for diabetic macular edema (DME) and diabetic retinopathy (DR) because c-Raf is a downstream signaling mediator for multiple growth factors responsible for DME and DR such as the vascular endothelial growth factor (VEGF), the insulinlike growth factor (IGF), the basic fibroblast growth factor (bFGF), and others (Grillone and Henry 2008). iCo-007 is a second generation ASO targeting c-Raf kinase that has been discovered by ISIS Pharmaceuticals Inc. (Carlsbad, California, USA). In pig models of branch vein occlusion 
and mouse models of laser induced choroidal neovascularization the ASO caused inhibition of c-Raf expression and improvement in the neovascularization severity score (Danis et al. 2003). iCo-007 was licensed to iCo Therapeutics Inc. (Vancouver, British Columbia, Canada) in 2005 and since then it has been in an open label, dose escalating Phase I clinical trial in patients with DME. In a study, the drug was administered as a single intravitreous injection to patients with DME which did not respond to other treatments. The drug did not show any serious adverse events and lead to reduction of macular edema in some patients (Hnik et al. 2009). Those results suggest that iCo-007 might be an effective treatment for clinical diseases associated with neovascularization and leakage (other than DME and DR), viral infections, intraocular inflammation and glaucoma.

\subsection{Two Locked Nucleic Acid-modified ASOs for the Cure of T2D}

The peroxisome proliferator-activated receptor (PPAR) $\gamma$ and the proteintyrosine phosphatase (PTP)-1B are validated targets for T2D and thirdgeneration ASOs have been developed against them. PPAR $\gamma$ is a component of the nuclear hormone receptor superfamily and plays an important role in inflammation, cell cycle regulation and differentiation, and glucose homeostasis. Treatment of wild-type mice with a PPAR $\gamma$ antagonist has been reported to prevent high fat diet-induced obesity, insulin resistance, and T2D. Tachibana et al. designed a third-generation locked nucleic acid targeting the translation initiation site of human PPAR $\gamma$ mRNA and showed its inhibitory effect on two human cell lines (THP-1 and HCT116) (Tachibana et al. 2007). PTP-1B negatively regulates insulin receptor in insulinresponsive tissues (such as adipose, liver and muscle). ISIS Pharmaceuticals Inc. has been developing a second-generation ASOs (ISIS113715) to inhibit expression of PTP-1B. In preclinical studies ISIS113715 was able to normalize the plasma glucose levels in treated $o b / o b$ and $d b / d b$ mice and improved insulin sensitivity and adiponectin concentration in primates (Swarbrick et al. 2009). Recent phase II data showed that T2D patients well tolerated the ASOs and showed statistically significant reduction in several indices of blood glucose control. Furthermore, Koizumi et al. developed a thirdgeneration locked nucleic acid isosequencial of ISIS113715 and shown to have enhanced in vivo efficacy in treated $d b / d b$ mice respect to ISIS113715 (Koizumi et al. 2007).

\subsection{Oligonucleotide-loaded PROMAXX ${ }^{\circledR}$ Microspheres, a Diabetes Suppressive Vaccine}

PROMAXX ${ }^{\circledR}$ microspheres technology has been developed by Epic Therapeutics Inc. The microspheres are fabricated through a phase- 
separation process that takes place in an aqueous environment at mild temperatures and enables the encapsulization of biomacromolecules. The size of microspheres can be tuned by adjusting the conditions of the process (ionic strength, $\mathrm{pH}$, rate of cooling, etc.) and the release of entrapped molecules can be programmed over times spanning from hours to weeks, thus drastically reducing the need for injections. Epic Therapeutics Inc. has characterized PROMAXX ${ }^{\circledR}$ formulations for pulmonary delivery of several drugs such as insulin, alpha- 1 anti-trypsin, human growth hormone, etc. Phillips et al. used PROMAXX ${ }^{\circledR}$ microspheres to deliver ASOs into dendritic cells (DC) to down-regulate the co-stimulatory receptors CD80/CD86 and CD40 (immature state). Several reports have shown that the exogenous administration of DC in an immature state efficiently prevents and suppresses diabetes in NOD mice. However this technology has serious logistical limitations due to the fact that the clinic (where the patients are treated) is often far away from the facilities where the DC are engineered. In an effort to avoid those limitations, Phillips et al. use PROMAXX ${ }^{\circledR}$ microspheres to elicit immature state in DC directly in vivo. The microspheres were safe and neutral on DC maturation and, when loaded with ASOs against co-stimulatory receptors, able to prevent T1D and reverse hyperglycemia (Phillips et al. 2008). These results suggest that the ASO-microsphere approach is feasible and can be easily applied for other autoimmune diseases.

\subsection{Oligonucleotide-conjugated Gold Nanoparticles, Novel Antisense Agents for T1D}

Gold nanoparticles (GNs) are widely used as nanocarriers because they are easy to synthesize in uniform and tunable size and can be subjected to a variety of chemical functionalizations. GNs can be densely functionalized with thiol-modified oligonucleotides, to yield particles that are stable under physiological conditions and can bind the complementary nucleic acids with very high affinity. Recently Rosi et al. investigated GNs functionalized with ASOs (GN-ASOs) (Rosi et al. 2006) and reported that they were readily uptaken by various cell types (RAW 264.7, HeLa, NIH$3 \mathrm{~T} 3$ and MDCK), did not affect cellular morphology and viability, and were not digested by intracellular nucleases up to 48 hours after internalization. GN-ASOs outperformed commercially available cationic liposomal delivery systems in a cell transfection system and their performance could be enhanced by increasing the number of ASOs decorating the GNs. Rink et al. successfully used GN-ASOs in vivo to enhance islet resistance after transplantation, and graft survival by targeting specific cytokine and chemokine receptors, pro apoptosis genes and second messenger signaling pathway molecules in $\beta$ cells. In a recently published work they showed 
that GN-ASOs were able to fully penetrate into all the cells of purified human islet (Rink et al. 2010). This was a remarkable achievement since pancreatic islets are compact three dimensional collections of cells whose core is usually unreachable by transfection agents such as viral vectors and cationic liposomes. The same group also observed that GN-ASOs did not negatively impact the viability and functionality of $\beta$ cells, since 1 ) conjugate-treated islets were able to secrete the same amount of insulin in response to glucose as untreated islets and 2) diabetic mice transplanted with treated islets were able to achieve euglycemia comparable to mice transplanted with untreated islets. Importantly the authors also showed that GNs conjugated with anti-eGFP ASOs were able to reduce the expression of eGFP in islets from transgenic MIP-eGFP mice that express eGFP under the control of the insulin promoter. These results suggest that GN-ASOs are a promising platform for the fabrication of ASO-based tools for modulating gene expression in vitro and in vivo.

\subsection{PEGylated Carbon Nanotubes as Cell-specific Delivery Systems for ASOs}

Our laboratory has been experimenting with carbon nanotubes (NTs) functionalized with polyethylene glycol (PEG) in the effort to generate an efficient targeted delivery systems for ASOs. We and others functionalize NTs with PEG chains (Liu et al. 2009; Delogu et al. 2009) by adsorption of phospholipids terminated with activated PEG chains (DSPE-PEG) onto NTs. This is a non-covalent procedure based on ultrasonication, stepwise ultrafiltration and purification by ultrafiltration (PEGylated carbon nanotubes or PNTs) (Fig. 5). PNTs are excellent nanocarriers due to their physicochemical properties (multivalency, nanoscopic dimension,

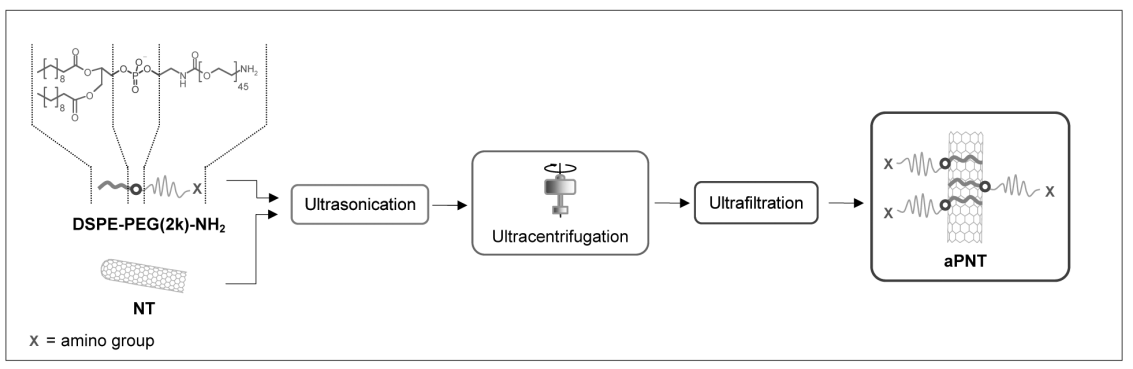

Fig. 5. Fabrication of functionalized PEGylated carbon nanotubes. Pristine (nonfunctionalized) carbon nanotubes (NTs) were ultrasonicated with phospholipids terminated with amino-functionalized 2-kDa mw PEG chains \{1,2-distearoyl-sn-glycero3-phosphoethanolamine- $N$-[amino(polyethylene glycol) $\left.{ }_{2000}\right]$ [DSPE-PEG(2k)- $\mathrm{NH}_{2}$ ], fractionated by stepwise ultracentrifugation to isolate short hydrophilic amino-terminated PEGylated NTs and purified by ultrafiltration to remove free phospholipids (aPNT).

Color image of this figure appears in the color plate section at the end of the book. 
and solubility under physiological conditions), low toxicity and excellent pharmacokinetic profile (Liu et al. 2009; Schipper et al. 2008). We focused on achieving PNT-based in vivo delivery of nanodrugs into $\mathrm{T}$ cells, which are key player in the pathogenesis of T1D. In a first report, we functionalized PNTs with monoclonal antibodies specific for receptors expressed on the T cell plasma membrane and a fusogenic polymer (PNT$\mathrm{mAb}-\mathrm{p}$ ), and intravenously injected them in mice (Cato et al. 2008). The PNT-mAb-p were found to be delivered in the cytoplasm of T cells with high specificity, thus suggesting that the fusogenic polymer aided the nanoparticles to escape from the lysosomal compartments. Recently, we used PNTs to deliver ASOs in Jurkat T cells and achieve knock-down of the protein tyrosine phosphate PTPN22, a pathogenic factor and drug target for T1D (Delogu et al. 2009; Bottini et al. 2006). PNTs were conjugated with anti-PTPN22 phosphorothioate-modified ASOs through a disulfide bond (PNT-ASO) and incubated with T cells in culture. The disulfide bond was intended to be cleaved once the nanoassemblies were into the lysosomal compartments and enable the release of the ASOs from the PNTs. An order of magnitude higher reduction of PTPN22 expression than that produced by electroporation was observed. Our results suggest that PNTs-conjugated ASOs could be exploited to achieve gene knockdown in T cell in vivo, a goal which is relevant for therapy of T1D and other $\mathrm{T}$ cell mediated human diseases.

\section{Applications to Areas of Health and Diseases}

Target-specific ASOs are potentially much easier to optimize and handle than chemical inhibitors. These features of ASOs make them appealing as a tool for scientists involved in studies of mechanism of disease and as potential drugs in the therapeutic arena. ASOs have promising applications in the diabetes field. A few ASOs are already performing well in T2D clinical trials. Unfortunately poor spontaneous cell penetration has been a major drawback of ASOs in biomedical studies and therapeutic experimentation. Nanotechnology-based approaches can significantly boost ASO cell permeability. The use of nanocarriers will further expand the range of the in vivo applications of ASOs to include for example immune modulation and beta cell protection-regeneration in the T1D field. Nanotech also can further enhance the performance/safety profile of ASOs by enabling tissue targeting (for example to metabolically-relevant organs) or conferring to the ASO the ability to selectively affect specific immune cell subpopulations which play positive or negative role in the pathogenesis of T1D. 


\section{Key Facts of Drug Delivery}

- ASOs represent a powerful therapeutic weapon for human diseases. However one of the major limitations of ASOs is their poor cellpermeability.

- The use of nanocarriers can improve the accumulation of ASOs in organs and tissues relevant to diabetes and their delivery into specific cell subpopulations.

- The multivalent structure of some nanocarriers can be exploited to improve the potency of ASOs by increasing the number of triggered receptors and/or of delivered ASOs per internalization event.

- Nanocarriers with finely tuned size and shape may trigger cellmediated endocytosis through normally internalization-impaired receptors and therefore expand the spectrum of the receptors that can be exploited for cell internalization.

\section{Dictionary}

c-Raf kinase: c-Raf kinase is a mitogen-activated protein (MAP) kinase kinase kinase (MAP3K), encoded in humans by the RAF1 gene and involved in cellular processes regulating differentiation, proliferation and apoptosis. Enzymes are proteins that catalyze chemical reactions. In enzymatic reactions, the molecules at the beginning of the process are called substrates, and the enzyme converts them into different molecules, called the products. Almost all processes in a biological cell need enzymes to occur at...c-Raf functions downstream of the Ras sub-family of membrane associated GTPases and regulates the activation of the dual specificity protein kinases MEK1 and MEK2, which, in turn, activate the serine/threonine specific protein kinases ERK1 and ERK2. The latter are important player in the control of the expression of genes involved in cell division, differentiation, migration and apoptosis.

Euglycemia: Euglycemia, also called normoglycemia, is the condition of having a normal level of sugar in the blood.

GFP: The green fluorescent protein (GFP) is a small protein (238 aminoacids, approximately $30 \mathrm{kDa}$ ) that emits in the green range of the visible light if excited by blue light. It was first isolated from the jellyfish Victoria Aequorea. The GFP gene has been successfully introduced in cells, bacteria, fungi and in living animals to label specific proteins whose biological processes can be then followed by simple fluorescent microscopy. Several mutants of GFP have been engineered to change its spectral properties. In particular, the enhanced GFP (eGFP) is a one-point mutant that exhibits increased fluorescence and photostability respect to GFP. 
Kinase: A kinase is an enzyme that transfers a phosphate group from a donor molecule (for instance ATP) to its substrate. This process is called phosphorylation. The inverse process, the removal of a phosphate group, is carried out by enzymes called phophatases.

PTPN22: The protein tyrosine phosphatase (PTP) N22 is expressed only in white blood cells, where it negatively regulates the T cell receptor (TCR) signalling by cleaving activating phosphate groups from several critical TCR signalling mediators. A genetically encoded variant of PTPN22 carrying a single Arg to Trp aminoacid substitution in position 620 is a strong risk factor for T1D and other autoimmune diseases. Recent data showed that the Trp620 mutant of PTPN22 weaken signalling through the TCR. Small reductions of TCR signalling negatively affect immune tolerance through a variety of mechanisms. Given the increased activity of the autoimmune-predisposing PTPN22 variant, selective inhibition of PTPN22 in carriers of the mutant has been envisaged as an effective etiological therapy for autoimmune diseases in carriers of the Trp620 variant.

\section{Summary}

- Antisense oligonucleotides are short (typically 20bp in length) singlestranded deoxyribonucleotide analogues that hybridizes with the complementary mRNA via Watson-Crick base pairing and can block gene expression.

- Antisense oligonucleotides represent a powerful therapeutic weapon for diseases associated with dysregulated protein expression.

- Several chemical modifications of the phosphodiester bond and/or the ribose sugar have been identified which improve the pharmacokinetic profile and increase the potency of the antisense oligonucleotides.

- All the antisense oligonucleotide-based drugs under clinical investigations are administered in "free" form or encapsulated into cationic liposomes.

- A major issue for antisense oligonucleotide-based therapeutics is their poor cell-permeability.

- Nanotechnology-derived particles (nanocarriers) offer the possibility to target delivery of antisense oligonucleotides with high efficiency to their site of action.

- The use of nanocarriers for the targeted delivery of antisense oligonucleotides is still a young field and the choice of the optimal system can be complicated. 


\section{Abbreviations}

$\begin{array}{lrl}\text { ASOs } & : & \text { antisense oligonucleotides } \\ \text { bFGF } & : & \text { basic fibroblast growth factor } \\ \text { DC } & : & \text { dendritic cell } \\ \text { DTT } & : & \text { Dithiothreitol } \\ \text { eGFP } & : & \text { enhanced green fluorescent protein } \\ \text { EPR } & : & \text { enhanced permeability and retention effect } \\ \text { DME } & : & \text { diabetic macular edema } \\ \text { DNA } & : & \text { deoxyribonucleic acid } \\ \text { DR } & : & \text { diabetic retinopathy } \\ \text { FDA } & : & \text { food and drug administration } \\ \text { GN } & : & \text { gold nanoparticle } \\ \text { IGF } & : & \text { insulin-like growth factor } \\ \text { MAP } & : & \text { mitogen-activated protein } \\ \text { mRNA } & : & \text { messenger RNA } \\ \text { NTs } & : & \text { carbon nanotubes } \\ \text { PEG } & : & \text { polyethylene glycol } \\ \text { PNTs } & : & \text { PEGylated carbon nanotubes } \\ \text { PPAR } & : & \text { peroxisome proliferator-activated receptor } \\ \text { pre-mRNA } & \text { precursor mRNA } \\ \text { PTPN22 } & : & \text { protein tyrosine phosphate N22 } \\ \text { RES } & : & \text { reticulo endothelial system } \\ \text { RME } & : & \text { receptor mediated endocytosis } \\ \text { RNA } & : & \text { ribonucleic acid } \\ \text { RNase } & : & \text { ribonuclease } \\ \text { RT-PCR } & : & \text { real time polymerase chain reaction } \\ \text { siRNA } & : & \text { small interference RNA } \\ \text { TCR } & : & \text { T cell receptor } \\ \text { TLR } & : & \text { toll like receptor } \\ \text { T1D } & : & \text { type 1 diabetes } \\ \text { T2D } & : & \text { type 2 diabetes } \\ \text { VEGF } & : & \text { vascular endothelial growth factor } \\ & & \end{array}$

\section{References}

Bhat, B.G., H. Younis, J. Herrera, K. Palacio, B. Pascual, G. Hur, B. Jessen, K.M. Ogilvie and P.A. Rejto. 2007. Antisense inhibition of 11betahydroxysteroid dehydrogenase type 1 improves diabetes in a novel cortisone-induced diabetic KK mouse model. Biochem. Biophys. Res. Commun. 365: 740-5.

Bottini, N., T. Vang, F. Cucca and T. Mustelin. 2006. Role of PTPN22 in type 1 diabetes and other autoimmune diseases. Semin. Immunol. 18: 207-213. 
Brenner, B.M., W.M. Deen and C.R. Robertson. 1976. Determinants of glomerular filtration rate. Annu. Rev. Physiol. 38: 11-19.

Cato, M.H., F. D’Annibale, D.M. Mills, F. Cerignoli, M.I. Dawson, E. Bergamaschi, N. Bottini, A. Magrini, A. Bergamaschi, N. Rosato, R.C. Rickert, T. Mustelin and M. Bottini. 2008. Cell-type specific and cytoplasmic targeting of PEGylated carbon nanotube-based nanoassemblies. J. Nanosci. Nanotechnol. 8: 2259-69.

Chan, J.H.P., S. Lim and W.S.F. Wong. 2006. Antisense oligonucleotides: from design to therapeutic application. Clin. Exp. Pharmacol. Physiol. 33: 533-40.

Danis, R., M. Criswell, F. Orge, E. Wancewicz, K. Stecker, S. Henry and B. Monia. 2003. Intravitreous anti-raf-1 kinase antisense oligonucleotide as an angioinhibitory agent in porcine preretinal neovascularization. Curr. Eye Res. 26: $45-54$.

Delogu, L.G., A. Magrini, A. Bergamaschi, N. Rosato, M.I. Dawson, N. Bottini and M. Bottini. 2009. Conjugation of antisense oligonucleotides to PEGylated carbon nanotubes enables efficient knockdown of PTPN22 in T lymphocytes. Bioconjug. Chem. 20: 427-31.

DeLong, R.K., H. Yoo, S.K. Alahari, M. Fisher, S.M. Short, S.H. Kang, R. Kole, V. Janout, S.L. Regan and R.L. Juliano. 1999. Novel cationic amphiphiles as delivery agents for antisense oligonucleotides. Nucleic Acids Res. 27: 3334-41.

Grillone, L.R., and S.P. Henry. Potential therapeutics: applications of antisense oligonucleotides in ophthalmology. pp. 585-600. In: S.T. Crooke [eds.] 2008. Antisense drug technology: principles, strategies, and applications. CRC Press Taylor \& Francis Group, New York, USA.

Hnik, P., D.S. Boyer, L.R. Grillone, J.G. Clement, S.P. Henry and E.A. Green. 2009. Antisense oligonucleotide therapy in diabetic retinopathy. J. Diabetes Sci. Technol. 3: 924-30.

Jia, N., Q. Lian, H. Shen, C. Wang, X. Li and Z. Yang. 2007. Intracellular delivery of quantum dots tagged antisense oligodeoxynucleotides by functionalized multiwalled carbon nanotubes. Nano Lett. 7: 2976-80.

Jiang, W., B.Y.S. Kim, J.Y. Rutka and W.C.W. Chan. 2008. Nanoparticle-Mediated Cellular Response Is Size-Dependent. Nat. Nanotechnol. 3: 145-150.

Juliano, R.L. Biological barriers to nanocarrier-mediated delivery of therapeutic and imaging agents. pp. 263-278. In: C.M. Niemeyer, and C.A. Mirkin. [eds.] 2007. Nanobiotechnology II. Wiley-VCH, Weinheim, Germany.

Juliano, R.L., M.R. Alam, V. Dixit and H. Kang. 2008. Mechanisms and strategies for effective delivery of antisense and siRNA oligonucleotides. Nucleic Acids Res. 36: 4158-71.

Kang, H., M.R. Alam, V. Dixit, M. Fisher and R.L. Juliano. 2008. Cellular delivery and biological activity of antisense oligonucleotides conjugated to a targeted protein carrier. Bioconjug. Chem. 19: 2182-8.

Khan, A., M. Benboubetra, P.Z. Sayyed, K.W. Ng, S. Fox, G. Beck, I.F. Benter and S. Akhtar. 2004. Sustained polymeric delivery of gene silencing antisense ODNs, siRNA, DNAzymes and ribozymes: in vitro and in vivo studies. Drug Target 12: 393-404.

Koizumi, M., M. Takagi-Sato, R. Okuyama, K. Araki, W. Sun and D. Nakai. 2007. In vivo antisense activity of ENA oligonucleotides targeting PTP1B mRNA in 
comparison of that of 2'-MOE-modified oligonucleotides. Nucleic Acids Symp. Ser. 51: 111-2.

Kostarelos, K., L. Lacerda, G. Pastorin, W. Wu, S. Wieckowski, J. Luangsivilay, S. Godefroy, D. Pantarotto, J.P. Briand, S. Muller, M. Prato and A. Bianco. 2007. Cellular uptake of functionalized carbon nanotubes is independent of functional group and cell type. Nat. Nanotechnol. 2: 108-13.

Liang, Y., M.C. Osborne, B.P. Monia, S. Bhanot, L.M. Watts, P. She, S.O. DeCarlo, X. Chen and K. Demarest. 2005. Antisense oligonucleotides targeted against glucocorticoid receptor reduce hepatic glucose production and ameliorate hyperglycemia in diabetic mice. Metabolism 54: 848-55.

Liu, Z., S. Tabakman, K. Welsher and H. Dai. 2009. Carbon Nanotubes in Biology and Medicine: In vitro and in vivo Detection, Imaging and Drug Delivery. Nano Res. 2: 85-120.

Mosser, D.M., and J.P. Edwards. 2008. Exploring the full spectrum of macrophage activation. Nat. Rev. Immunol. 8: 958-69.

Phillips, B., K. Nylander, J. Harnaha, J. Machen, R. Lakomy, A. Styche, K. Gillis, L. Brown, D. Lafreniere, M. Gallo, J. Knox, K. Hogeland, M. Trucco and N. Giannoukakis. 2008. A microsphere-based vaccine prevents and reverses new-onset autoimmune diabetes. Diabetes 57: 1544-55.

Rink, J.S., K.M. McMahon, X. Chen, C.A. Mirkin, C.S. Thaxton and D.B. Kaufman. 2010. Transfection of pancreatic islets using polyvalent DNA-functionalized gold nanoparticles. Surgery 148: 335-45.

Rosi, N.L., D.A. Giljohann, C.S. Thaxton, A.K. Lytton-Jean, M.S. Han and C.A. Mirkin. 2006. Oligonucleotide-modified gold nanoparticles for intracellular gene regulation. Science 312: 1027-30.

Scherphof, G.L. In vivo behavior of liposomes: Interactions with the mononuclear phagocyte system and implications for drug targeting. pp. 285-300. In: R.L. Juliano. [eds.] 1991. Targeted Drug Delivery. Springer, New York, USA.

Schipper, M.L., N. Nakayama-Ratchford, C.R. Davis, N.W. Kam, P. Chu, Z. Liu, X. Sun, H. Dai and S.S. Gambhir. 2008. A pilot toxicology study of single-walled carbon nanotubes in a small sample of mice. Nat. Nanotechnol. 3: 216-21.

Swarbrick, M.M., P.J. Havel, A.A. Levin, A.A. Bremer, K.L. Stanhope, M. Butler, S.L. Booten, J.L. Graham, R.A. McKay, S.F. Murray, L.M. Watts, B.P. Monia and S. Bhanot. 2009. Inhibition of protein tyrosine phosphatase-1B with antisense oligonucleotides improves insulin sensitivity and increases adiponectin concentrations in monkeys. Endocrinology 150: 1670-9.

Tachibana, K., T. Katayama, C. Ueda, M. Sumitomo, M. Tagami, K. Ishimoto, D. Yamasaki, T. Tanaka, T. Hamakubo, J. Sakai, T. Kodama, S. Obika, T. Imanishi and T. Doi. 2007. Antisense activity of $2^{\prime}, 4^{\prime}$-BNA targeted to PPAR gamma in THP-1 and HCT116 cells. Nucleic Acids Symp. Ser. 51: 441-2.

Yoo, H., and R.L. Juliano. 2000. Enhanced delivery of antisense oligonucleotides with fluorophore-conjugated PAMAM dendrimers. Nucleic Acids Res. 28: 4225-31.

Waring, J.F., R. Ciurlionis, J.E. Clampit, S. Morgan, R.J. Gum, R.A. Jolly, P. Kroeger, L. Frost, J. Trevillyan, B.A. Zinker, M. Jirousek, R.G. Ulrich and C.M. Rondinone. 2003. PTP1B antisense-treated mice show regulation of genes involved in lipogenesis in liver and fat. Mol. Cell Endocrinol. 203: 155-68. 
84 Nanotechnology and Nanomedicine in Diabetes 